\title{
Modeling microdebrider-mediated ophthalmic damage: a word of caution in endoscopic sinus surgery*
}

Cameron P. Worden', Carly A. Clark', Anna K. Senior', Rodney J. Schlosser², Adam J. Kimple', Brent A. Senior'

' Department of Otolaryngology-Head and Neck Surgery, University of North Carolina at Chapel Hill, Chapel Hill, NC, USA

2 Division of Rhinology and Sinus Surgery, Department of Otolaryngology-Head and Neck Surgery, Medical University of South Carolina, Charleston, SC, USA
Rhinology Online, Vol 2: 44 - 49, 2019

http://doi.org/10.4193/RHINOL/19.004

*Received for publication:

January 31, 2019

Accepted: February 24, 2019

Published: March 7, 2019

\begin{abstract}
Background: The microdebrider has become nearly universal in the treatment of sinonasal pathology; however, recent case reports have demonstrated the potential for major ophthalmic complications. The goal of this study was twofold: 1) determine the anatomical proximity of ophthalmic structures to the paranasal sinuses, and 2) assess the timeframe at which ophthalmic injury may occur with the use of a microdebrider during FESS utilizing a cadaveric model.
\end{abstract}

Methodology: Computed tomography scans from 50 patients were accessed retrospectively. The distances between the lamina papyracea (LP) and orbital structures were determined at varying depths. Seven cadavers (14 sides) were studied using three microdebrider systems operated by otolaryngology residents. Following removal of a window of LP, the time from activation of the microdebrider on the periorbita until transection of the medial rectus (MR), optic nerve (ON), and to aspiration of the globe were measured.

Results: The mean distance between the LP and MR at the level of the anterior aspect of the anterior ethmoid and basal lamella were $3.59 \pm 1.2 \mathrm{~mm}$ and $1.5 \pm 0.8 \mathrm{~mm}$, respectively. The mean distance between the LP and ON at the level of the basal lamella was $8.1 \pm 2.1 \mathrm{~mm}$. Mean transection times for the MR and ON were $13.4 \pm 7.3$ seconds and $37.3 \pm 9.2$ seconds, respectively, with minimum times of 4 seconds and 26 seconds.

Conclusions: The proximity of orbital structures to the paranasal sinuses and the rapidity of ophthalmic damage following violation of the periorbita reaffirms the need for cautious use of the microdebrider during FESS.

Key words: microdebrider, endoscopic sinus surgery, ophthalmic complications, cadaver, lamina papyracea

\section{Introduction}

Chronic rhinosinusitis (CRS) is a heterogeneous disease characterized by long-term sinonasal mucosa inflammation producing symptoms of nasal congestion and drainage, facial pressure, and hyposmia ${ }^{(1)}$. CRS is estimated to impact approximately $13 \%$ of the adult US population ${ }^{(2)}$ and pose an annual national health care cost upwards of $\$ 8.6$ billion dollars ${ }^{(3)}$. Introduced to the US in 1985, functional endoscopic sinus surgery (FESS) represents the current standard surgical intervention for CRS (4). Utilizing minimally invasive endoscopic techniques, FESS enhances mu- cociliary clearance through the enlargement of paranasal sinus ostia and the debridement of underlying diseased mucosa in an effort to improve sinonasal ventilation.

Over the past two decades, powered instruments have been a major technological advancement in FESS ${ }^{(5)}$. The microdebrider, which utilizes a vacuum rotatory design to precisely target and resect diseased mucosa ${ }^{(6)}$, has been shown to reduce intraoperative blood loss and surgery duration while promoting faster postoperative healing ${ }^{(7)}$. Early in-vitro studies advocated for the safety of microdebrider use in FESS, especially around delicate 
structures located in the paranasal sinuses ${ }^{(8)}$. However, this concept has been challenged as evidenced by numerous case studies that have shown that, when misplaced, the indiscriminate aspirating function and limited tactile feedback of the microdebrider can lead to major surgical complications ${ }^{\text {(9-12). }}$ The thin lamina papyracea (LP) separates orbital contents from the lateral aspect of the ethmoidal labyrinth. If this delicate bony plate is penetrated by the microdebrider during FESS, severe ophthalmic complications such as retrobulbar hematoma, enophthalmos, and blindness can occur rapidly ${ }^{(13)}$. Additionally, the medial rectus (MR) and inferior rectus muscles are at an increased risk of injury due to their intimacy with the LP, and injury to these extraocular muscles can result in permanent diplopia. Recent case reports have highlighted the potential for optic nerve (ON) injury ${ }^{(11)}$ and even enucleation ${ }^{(12)}$ as potential complications of microdebrider use during FESS. While knowledge of sinonasal anatomy, surgical experience, proper technique, and preoperative preparation are critical to reducing the incidence of iatrogenic injury during FESS, severe complications can occur even in the hands of the most experienced surgeon ${ }^{(14)}$.

The goal of this study was twofold: 1) retrospectively analyze patient computed tomography (CT) imaging to determine the anatomical proximity of ophthalmic structures to the paranasal sinuses, and 2) assess the timeframe at which ophthalmic injury may occur with the use of a microdebrider during FESS utilizing a cadaveric model. To our knowledge, no previous study has quantitatively assessed the rate of orbital structure damage from microdebrider use in a cadaveric model. Knowledge gained from this study will be influential in guiding endoscopic surgical decision-making and reinforcing the necessary respect of the use of powered instruments during sinus surgery.

\section{Materials and Methods}

\section{CT orbital measurements}

Following Institutional Review Board approval from the University of North Carolina, records from 50 patients seen in June 2018 with previous sinus CT imaging without contrast were accessed retrospectively for this study. From axial plane slices, the distance between the LP and MR at the level of the anterior aspect of the anterior ethmoid and basal lamella were measured for each patient (Figure 1). Additionally, the distance between the LP and the ON was determined at the level of the basal lamella (Figure 1). Finally, the angle between the optic nerve and the nasal midline was determined from the angle generated between two vectors - one vector along the nasal midline to the tip of the nose and the other vector from the tip of the nose to the emergence optic nerve from the globe (Figure 1).

\section{Cadaveric dissections}

All dissections occurred in conjunction with the Southern States Rhinology Foundation course at the Medical University of South

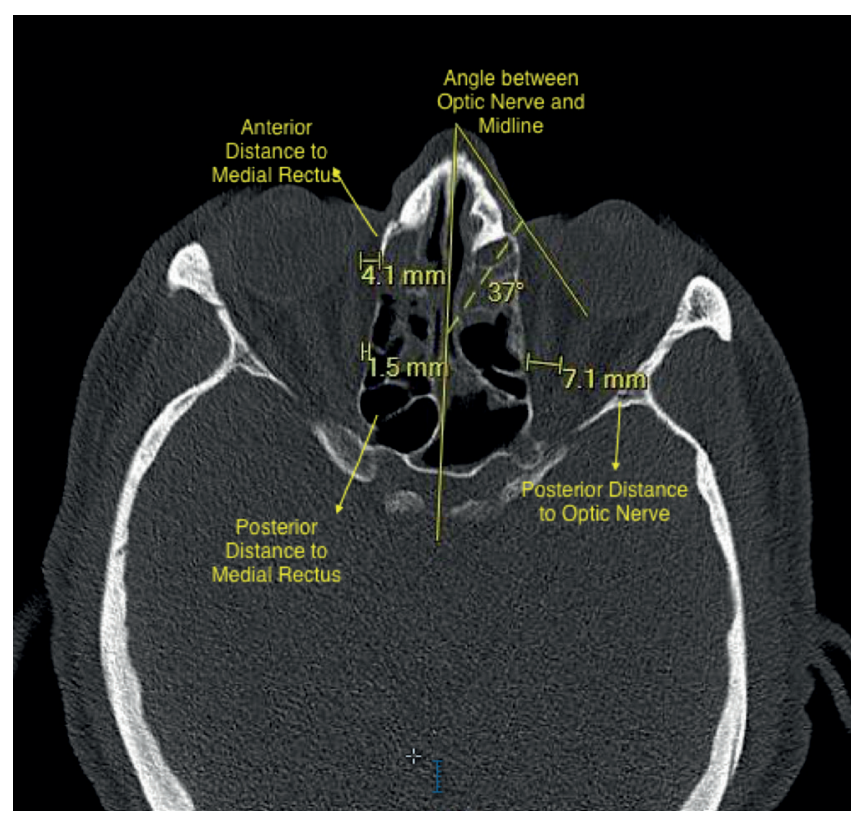

Figure 1. Axial CT measurements of the distance from the lamina papyracea to various orbital structures and the angle between optic nerve and the nasal midline.

\section{Carolina.}

Seven fresh, unembalmed cadavers were obtained for experimental use, for a total of 14 sides. All cadavers were positioned supine. Fourteen otolaryngology residents ranging from PGY 2-5 from two academic institutions (University of North Carolina and Medical University of South Carolina) were split into seven groups of two for dissection. One resident in the group of two dissected one side, while the other resident in the same group dissected the other side. All residents were operating righthanded.

Three separate microdebrider systems were used: Medtronic M5 Straightshot ${ }^{\circledR}$ with an IPC control unit, Karl Storz DrillCut-X II ${ }^{\circledR}$ with an UNIDRIVE ${ }^{\circledR}$ S III ECO/ENT/NEURO motor control unit, and the Entellus Medical Shaver System ${ }^{\oplus}$. All microdebriders utilized a $4 \mathrm{~mm}$ straight blade and were programmed to operate on oscillation mode with a rotational speed of $5000 \mathrm{rpm}$ and $5 \mathrm{cc} / \mathrm{min}$ of continuous irrigation and were attached identical standard lab suction. All microdebrider motor units, handpieces, foot pedals, and blades were donated from participating vendors for experimental use. Each group utilized only one microdebrider system for the entire dissection.

A complete ethmoidectomy and sphenoidotomy were performed bilaterally to expose the lamina papyracea on either side of the cadaver. Slight pressure was then placed on the external eye to test the integrity of the LP. Prolapse of orbital fat into the nasal cavity was not observed in any dissection group. Subsequently, an approximate $2 \mathrm{~cm} \times 2 \mathrm{~cm}$ square window of LP centered on the basal lamella was removed to expose, but not violate, the underlying periorbita. The microdebrider blade 


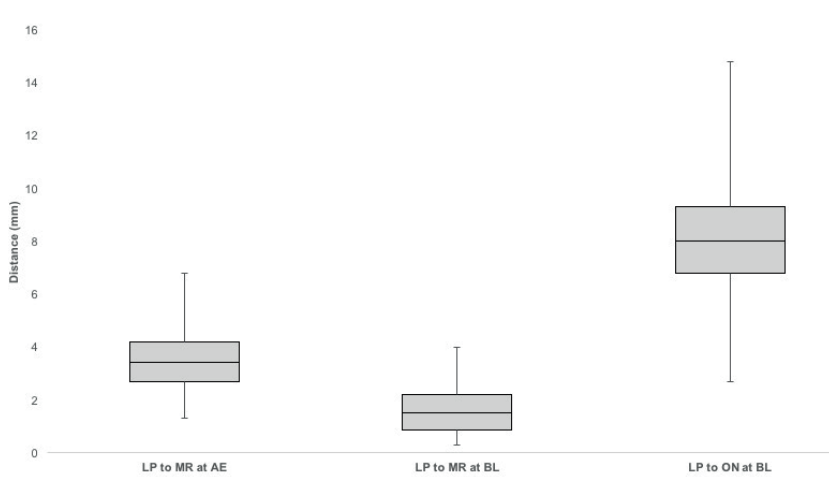

Figure 2. Distance from the lamina papyracea (LP) to medial rectus (MR) and optic nerve (ON) at depths of the anterior ethmoid (AE) and basal lamella (BL). Shown is a box-and-whisker plot for each measurement point. Total sides for each measurement point, $\mathrm{N}=100$. All measurements reported in millimeters $(\mathrm{mm})$.

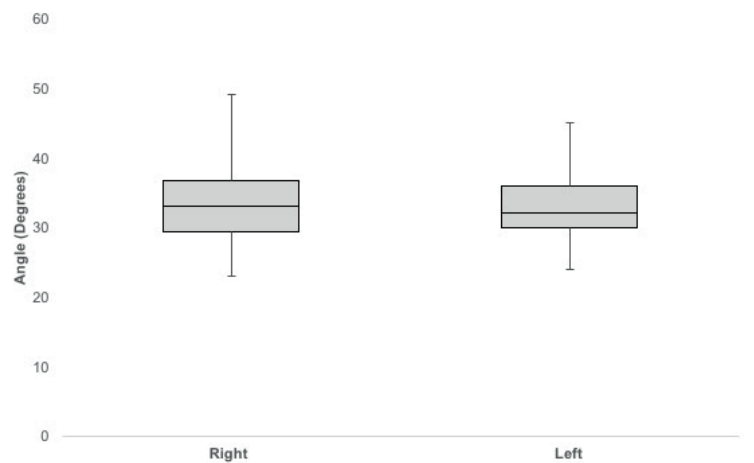

Figure 3. Angle between the optic nerve and the nasal midline. Shown is a box-and-whisker plot for the right and left side measurement points. Total sides for each measurement point, $\mathrm{N}=50$. All measurements reported in degrees. tip was then placed on the periorbita and activated. Time from activation of the microdebrider on the periorbita until complete transection of the MR and ON, and to perforation and aspiration of the globe were documented for each dissection group (Table 1). Transection and aspiration of orbital structures were visualized via nasal endoscopy. All times were measured using a standard stopwatch by the senior investigator (BAS).

\section{Results}

CT measurements of orbital structure proximity to the paranasal sinuses

The mean distance between the LP and MR at the level of the anterior aspect of the anterior ethmoid was determined to be $3.6 \mathrm{~mm}$ (S.D. 1.2mm; range 1.3-6.8 mm) (Figure 2). The mean distance between the LP and MR at the level of the basal lamella was $1.5 \mathrm{~mm}$ (S.D. $0.8 \mathrm{~mm}$; range $0.3-4.0 \mathrm{~mm}$ ) (Figure 2). The mean distance between the LP and ON at the level of the basal lamella was $8.1 \mathrm{~mm}$ (S.D. 2.1 mm; range 2.7-14.8 mm) (Figure 2). The mean angle between the optic nerve and the nasal midline was determined to be 33.2 degrees (S.D. 5.4 degrees; range 23-49 $\mathrm{mm}$ ) and 33.1 degrees (S.D. 4.6 degrees; range $24-45 \mathrm{~mm}$ ) on the right and left sides, respectively (Figure 3).

Timeline of microdebrider-mediated ophthalmic structure injury in a cadaveric model

The fastest time for complete transection of the MR was four seconds utilizing an Entellus shaver system (Table 1). The fastest time for complete transection of the ON was 26 seconds utilizing a Medtronic M5 Straightshot (Table 1). The mean times for MR and ON transection were $13.4 \pm 7.3$ seconds and $37.3 \pm 9.2$ seconds, respectively ( $\mathrm{N}=14$ sides). The globe was most rapidly perforated and aspirated in 47 seconds with an Entellus Medical Shaver System (Table 1). The mean time for globe perforation and aspiration was $68.3 \pm 14.2$ seconds ( $N=14$ sides).

\section{Discussion}

We performed an anatomic study using CT scans to determine the proximity of ophthalmic structures to the paranasal sinuses. Additionally, we simulated the timeline of potential ophthalmic complications resulting from microdebrider misplacement during FESS utilizing a cadaveric model. It is important to realize that while the microdebrider has revolutionized endoscopic sinus surgery by providing real-time, precise resection of diseased mucosa in a confined surgical field, the potential for rapid surgical complications must be viewed as an ever-present concern. As the microdebrider offers minimal tactile feedback in addition to accelerated tissue resection speeds as compared to conventional instrumentation ${ }^{(9)}$, the knowledge of anatomical landmarks and proper surgical technique become ever more critical in preventing adverse outcomes.

Retrospective CT measurements showed a mean distance from the LP to the MR at the level of the basal lamella of just $1.5 \pm$ $0.8 \mathrm{~mm}$. While the mean distance from the LP to the MR increased in a posterior to anterior fashion from the level of the basal lamella to the anterior ethmoid, a cautious approach to utilizing the microdebrider during ethmoidectomy is paramount. Cadaveric dissections displayed similar results. By placing the tip of the microdebrider on an exposed portion of periorbita, the microdebrider was able to completely transect the MR in one dissection group in just four seconds upon activation. Across all 14 sides, the mean time to MR transection time was comparable at 13.4 seconds. It is therefore conceivable that a simple lapse in concentration of the surgeon while utilizing the microdebrider during an ethmoidectomy could lead to permanent diplopia for a patient. This concept has been well documented in numerous reports of iatrogenic extraocular muscle injury resulting from inadvertent microdebrider placement during routine FESS $(10,15,16)$. If ophthalmic damage is suspected intraoperatively, the surgeon should perform the bulb press test to determine if the lamina 
Table 1. Timeline of microdebrider-mediated orbital structure injury in a cadaveric model ${ }^{a}$

\begin{tabular}{|c|c|c|c|c|c|c|c|}
\hline Group & $\begin{array}{c}\text { Microdebrider } \\
\text { system }\end{array}$ & MR & $\begin{array}{c}\text { Right } \\
\text { ON }\end{array}$ & Globe & MR & $\begin{array}{l}\text { Left } \\
\text { ON }\end{array}$ & Globe \\
\hline 1 & Medtronic & 15 & 32 & 85 & 14 & 35 & 74 \\
\hline 2 & Karl Storz & 8 & 51 & 54 & 8 & 27 & 104 \\
\hline 3 & Medtronic & 11 & 43 & 76 & 29 & 52 & 61 \\
\hline 4 & Karl Storz & 8 & 29 & 66 & 19 & 31 & 64 \\
\hline 5 & Medtronic & 6 & 26 & 61 & 16 & 52 & 68 \\
\hline 6 & Entellus & 26 & 42 & 65 & 4 & 35 & 74 \\
\hline 7 & Entellus & 14 & 35 & 57 & 9 & 32 & 47 \\
\hline $\begin{array}{l}\text { Mean } \pm S D \\
\quad(n=7)\end{array}$ & -- & $12.6 \pm 6.8$ & $36.9 \pm 8.9$ & $66.3 \pm 10.9$ & $14.1 \pm 8.3$ & $37.7 \pm 10.1$ & $70.3 \pm 17.5$ \\
\hline
\end{tabular}

a. All data reported in seconds.

papyracea has been violated. Any prolapse of orbital fat into the nasal cavity should be cause for immediate cessation of surgical activity in that area.

ON injury is a rare complication of FESS due to either retrobulbar hematoma or direct insult ${ }^{(14,17)}$. Retrospective $C T$ measurements showed a mean distance of just $8.1 \pm 2.1 \mathrm{~mm}$ from the LP to the $\mathrm{ON}$ at the level of the basal lamella. While the fastest and mean ON transection times across all seven dissection groups were 26 seconds and $37.3 \pm 9.2$ seconds, respectively, these data reinforce a similar cautious reminder of the rapidity of orbital damage following violation of the LP. If the periorbita is breached, a combination of surgical inexperience in addition to accelerated tissue resection speeds and limited tactile feedback offered by the microdebrider can make prompt detection of adverse complications difficult. Thus, continuous vigilance during tissue resection must remain a preeminent concern.

Finally, globe perforation and aspiration times were measured as to outline the timeline for enucleation as a potential surgical complication of microdebrider use, as evidenced by a recently published case report ${ }^{(12)}$. With one dissection group achieving complete enucleation in less than one minute (47 seconds), the capabilities of the microdebrider and powered instruments, as a whole, must be appreciated and respected. With the expansion in the customization of blade configurations, rotational velocity, and aspiration speeds, microdebriders have been continually expanding their role over the previous years. As microdebrider-meditated tissue resection efficacy increases, the balance between expanded functionality and the increased incidence of adverse outcomes will consequently become ever narrower. Additionally, as the scope of endoscopic sinus surgery continues to expand into office-based procedures, the risk of severe surgical complications from microdebrider use must be reevaluated to ensure patient safety in the setting of the clinic. While preliminary safety studies of the newly introduced clinic- oriented, vacuum-powered microdebrider have been promising ${ }^{(18,19)}$, the incidences of severe complications associated with its use have not been fully elucidated due to the limited time frame of analysis of these studies.

While the timeline for other potential ophthalmic complications such as orbital subcutaneous emphysema, nasolacrimal duct injury, periorbital ecchymosis, inferior rectus muscle injury, and enopthalamos were not measured in the cadaveric portion of this study, the severity of these adverse outcomes must also be appreciated. Notably, retrobulbar hematoma from injury to the anterior ethmoid artery, via the microdebrider, and subsequent retraction into the orbit has been illustrated in numerous case reports ${ }^{(14,20,21)}$. Due to the confined compartment of the orbit, an acute increase in volume can lead to the rapid build-up of intraocular pressure and progressive ophthalmic complications. Additionally, as the superior oblique muscle is often located in close proximity, attempts to ligate the retracted anterior ethmoid artery can result in permanent diplopia. If intraocular pressure rises above $40 \mathrm{mmHg}^{(21)}$, an immediate lateral canthotomy and cantholysis to decompress the orbit and consultation with an ophthalmologist are indicated.

While our preliminary analyses showed that, on average, rightsided microdebrider-mediated ophthalmic injury occurred more rapidly than left-sided ophthalmic injury, when utilizing only right-handed residents, the magnitude of the differences in Table 1 did not align with the traditional teaching and literature (22). One explanation is that due to the limited sample size of the study, we were not able to properly assess this observation and prove statistical significance. Future studies with appropriate power investigating this trend would be a potential fruitful avenue to pursue, as rigorously designed experiments investigating the rate of injury on side of approach in both right- and left-handed surgeons are lacking. Additionally, future powered studies that expanded on the investigation between the brand 
of microdebrider used and rate of ophthalmic structure injury would be another potentially insightful avenue to pursue. A potential limitation of this study is that utilizing otolaryngology residents at various levels of training, with subsequent differences in microdebrider operational experience, could help to explain some of the discrepancies seen in Table 1. Additionally, anatomical differences in the thickness of the periorbita and associated tissue of the cadavers cannot be discounted as potentially cofounding elements in the data analysis. However, we believe that the simplistic and regimented study design precludes differences in tissue resection speeds based on surgical experience from emerging. Future studies may be warranted in order to more rigorously outline the extent and timeline of ophthalmic complications from microdebrider use in order to guide endoscopic surgical decision-making in both the hospital and clinical domains.

\section{Acknowledgement}

Financial Conflict of Interest Disclosure: The project described was supported by the National Center for Advancing Translational Sciences, National Institutes of Health, through Grant KL2TR002490 to AJK. The content is solely the responsibility of the authors and does not necessarily represent the official views of the NIH.

\section{Authorship contribution}

CPW: Assistance with data gathering, data compiling, data analysis, and manuscript writing; CAC: Assistance with data gathering, data compiling, data analysis, and manuscript writing; AKS: Assistance with data gathering; RJS: Assistance with cadaver procurement and data gathering; AJK: Assistance with data compiling, data analysis, and manuscript writing; BAS: Assistance with cadaver procurement, data gathering, data compiling, data analysis, and manuscript writing.

\section{Conflict of interest}

The authors have no conflicts of interest to disclose.

\section{Ethics approval and consent to participate}

Not applicable.

\section{Consent for publication}

Not applicable

\author{
Availability of data and materials \\ Not applicable.
}

\section{Funding}

Financial Conflict of Interest Disclosure: The project described was supported by the National Center for Advancing Translational Sciences, National Institutes of Health, through Grant KL2TR002490 to AJK. The content is solely the responsibility of the authors and does not necessarily represent the official views of the $\mathrm{NIH}$.

\section{References}

1. Orlandi RR, Kingdom TT, Hwang PH, et al. International Consensus Statement on Allergy and Rhinology: Rhinosinusitis. International forum of allergy \& rhinology. 2016; 6 Suppl 1:S22-209.

2. Pleis JR, Lucas JW, Ward BW. Summary health statistics for U.S. adults: National Health Interview Survey, 2008. Vital and Health Statistics Series 10, Data from the National Health Survey. 2009; (242):1-157.

3. Bhattacharyya N. Incremental health care utilization and expenditures for chronic rhinosinusitis in the United States. The Annals of Otology, Rhinology, and Laryngology. 2011; 120(7):423-427.

4. Senior BA, Kennedy DW, Tanabodee $J$, et al. Long-term Results of Functional Endoscopic Sinus Surgery. Laryngoscope. 1998; 108(2):151-157.

5. Hackman TG, Ferguson BJ. Powered instru mentation and tissue effects in the nose and paranasal sinuses. Current Opinion in Otolaryngology \& Head and Neck Surgery. 2005; 13(1):22-26.

6. Setliff $\mathrm{RC}, 3 \mathrm{rd}$. The hummer: a remedy for apprehension in functional endoscopic sinus surgery. Otolaryngol Clin North Am. 1996; 29(1):95-104.
7. Ghera B, Munjal M, Chopra H. Comparative study of conventional versus microdebrider assisted endoscopic sinus surgery in sinonasal polyposis. Int J Otorhinolaryngol Head Neck Surg. 2016 Oct; 2(4):197-204

8. Ferguson BJ, DiBiase PA, D'Amico F. Quantitative analysis of microdebriders used in endoscopic sinus surgery. American Journal of Otolaryngology. 1999; 20(5):294297

9. Tang D, Lobo BC, D'Anza B, et al. Advances in Microdebrider Technology: Improving Functionality and Expanding Utility. Otolaryngol Clin North Am. 2017; 50(3):589598.

10. Thacker NM, Velez FG, Demer JL, et al. Extraocular muscle damage associated with endoscopic sinus surgery: an ophthalmology perspective. American Journal of Rhinology. 2005; 19(4):400-405.

11. Vásquez LM, González-Candial M. Permanent Blindness after Endoscopic Sinus Surgery. Orbit. 2011; 30(2):108-110.

12. Chang JR, Grant MP, Merbs SL. Enucleation as Endoscopic Sinus Surgery Complication. JAMA Ophthalmology. 2015; 133(7):850852.

13. Hosemann W, Draf C. Danger points, complications and medico-legal aspects in endoscopic sinus surgery. GMS Current Topics in Otorhinolaryngology, Head and Neck Surgery. 2013; 12:Doc06.

14. Stankiewicz JA, Lal D, Connor M, et al. Complications in endoscopic sinus surgery for chronic rhinosinusitis. The Laryngoscope. 2011; 121: 2684-2701.

15. Neuhaus RW. Orbital Complications Secondary to Endoscopic Sinus Surgery. Ophthalmology. 1990; 97(11):1512-1518.

16. Sohn JH, Hong SD, Kim JH, et al. Extraocular muscle injury during endoscopic sinus surgery: a series of 10 cases at a single center. Rhinology. 2014; 52(3):238-245.

17. Stankiewicz JA. Blindness and intranasal endoscopic ethmoidectomy: prevention and management. Otolaryngology--Head and Neck Surgery. 1989; 101(3):320-329.

18. Gan EC, Habib AR, Hathorn I, et al. The efficacy and safety of an office-based polypectomy with a vacuum-powered microdebrider. International Forum of Allergy \& Rhinology. 2013; 3(11):890-895.

19. Scott JR, Sowerby LJ, Rotenberg BW. Officebased Rhinologic Surgery: A Modern Experience with Operative Techniques Under Local Anesthetic. American Journal of Rhinology \& Allergy. 2017; 31(2):135-138.

20. Thompson RF, Gluckman JL, Kulwin D, 
Savoury L. Orbital hemorrhage during ethmoid sinus surgery. Otolaryngology-head and neck surgery. 1990; 102(1):45-50.

21. Graham SM, Nerad JA. Orbital Complications in Endoscopic Sinus Surgery Using Powered Instrumentation. The Laryngoscope. 2003; 113(5):874-878.

22. Dessi P, Castro F, Triglia JM, Zanaret M Cannoni M. Major complications of sinus surgery: a review of 1192 procedures. The Journal of laryngology and otology. 1994;108(3):212-215.
Brent A. Senior, MD

Department of OtolaryngologyHead and Neck Surgery University of North Carolina Memorial Hospitals 170 Manning Dr. Ground Floor Physician Office Building CB\#7070, Chapel Hill NC 27599 USA

Tel: +1-(984) 974-6484

Fax: +1(984)974-3499

E-mail: brent_senior@med.unc.edu

ISSN: 2589-5613 / (2019 The Author(s). This work is licensed under a Creative Commons Attribution 4.0 International License. The images or other third party material in this article are included in the article's Creative Commons license, unless indicated otherwise in the credit line; if the material is not included under the Creative Commons license, users will need to obtain permission from the license holder to reproduce the material. To view a copy of this license, visit http://creativecommons.org/licenses/by/4.0/ 